OPEN AcCESS Freely available online
www.banglajol.info/index.php/JSF
Journal of Science Foundation
July 2018, Vol. 16, No. 2, pp. $32-33$
ISSN (Print) 1728-7855
DOI: http://dx.doi.org/10.3329/jsf.v16i2.40865

\title{
Editorial
}

\section{Medication and Treatment Complexity of Epilepsy: Bangladesh Perspective}

\section{Mohammad Enayet Hussain ${ }^{1}$}

Epilepsy is a spectrum of neurological disorders which varies in manifestation, course, and prognosis. The most of the epilepsy patients need to take medications properly and follow a treatment plan over a period of time. The importance of adherence with AED therapy is well documented (Garnett, 2000). Yet factors that contribute to non-adherence, including the complexity of treatment regimens, have been difficult to quantify.

Current consensus guidelines for the use of antiepileptic drugs (AED) suggest that monotherapy is preferred (Karceski, Morrell, \& Carpenter, 2000), yet many patients require multiple medications to control their seizures. These more complex treatment regimens increase the burden of taking medications and are associated with lower medication adherence (Stone et al., 2001). Although investigators and clinicians generally determine medication complexity by summing the number of medications, the number of pills, the number of times per day each medicine is taken, or all of these, complexity has not been uniformly measured. Some investigators measure complexity by counting the number of medications; others use the number of doses taken per day (DiIorio, Faherty, \& Manteuffel, 1991; Graveley \& Oseasohn, 1991).

Other aspects of pill-taking that can add to complexity are often ignored by investigators and clinicians (Stone et al., 2001). These aspects include special instructions associated with medications (e.g., take on an empty stomach, take with food) and the administration actions required for taking the medicine (e.g., take half of the pill, crush pills).

In 1988, Kelley developed the Medication Complexity Index (MCI) to provide a uniform measure of medication complexity and expand the measure of complexity to include both special medication-taking instructions and actions. MCI originally developed for measuring complexity of medication regimens of the elderly (Kelley), was modified for epilepsy treatment regimens. This article describes the development and testing of the Epilepsy Medication and Treatment Complexity Index (EMTCI).

According to Kelley (1988), taking medications as prescribed requires that a person take the correct medications at the correct times in the correct dosage. It also requires that a person follow recommended instructions, such as taking the pills with or without food, and prepare medications according to specific directions. Medication complexity is defined by Kelley as the sum total of medications, doses, actions, and directions.

To measure medication complexity, Kelley (1988) developed a form to record information for each of these four components: number of medications, number of doses per day, mechanical actions, and additional directions. The information is converted into a numeric system by using a standardized coding scheme. One point is given for each time the medication is taken during the day and for each additional direction required. However, mechanical actions are weighted by the number of times a medication is taken per day. For example, a person taking one tablet of ranitidine twice per day receives a score of 2 . A person taking furosemide $30 \mathrm{mg}$ ( $1 \frac{1 / 2}{2}$ tablets) twice a day receives 2 points for twice-a-day administration plus an additional 2 points for the mechanical action of dividing a pill in half twice a day.

\footnotetext{
${ }^{1}$ Associate Professor, Department of Neurology, National Institute of Neurosciences \& Hospital, Sher-E-Bangla Nagar, Agargaon, Dhaka, Bangladesh; Email: enayetdmc@yahoo.com; Cell no.: +8801716215871
} 
To assess its stability over time, nurses completed the MCI for medication regimens twice in a 2-week period. Test-retest agreement ranged from 0.37 to 1.0, with higher agreement associated with the less complex regimens (Kelley, 1988). Validity was assessed by comparing the complexity rankings of medication regimens as determined by clinical experts to the scores on MCI. The correlation between expert rankings of complexity and MCI scores was 0.89 , providing evidence for criterion validity of the tool (Kelley).

The treatment of epilepsy is complicated and often fails to achieve the one main goal of treatment - the elimination of seizures. Far too often patients are treated with multiple drugs that lead to serious adverse events or drug-drug interactions and still have seizures that impair their ability to function. There is an everpresent need for newer, more effective agents with fewer side effects and less complicated drug-drug interactions. Clearly, there is a significant place for pharmacists in assuring appropriate treatment and monitoring is provided for epilepsy patients.

Since 2009, five, new antiepileptic (AEDs) have been approved by the US Food and Drug Administration (FDA) including vigabatrin, rufinamide, clobazam, ezogabine, and lacosamide. These drugs are all unique in their pharmacology and their indications, including atonic seizures, Lenox-Gastaut syndrome, infantile spasms, and add-on for partial epilepsy. On October 22, 2012, the FDA approved yet another agent for the treatment of partial-onset seizures in people with epilepsy - perampanel [Fycompa®, Eisai, Inc]. The search for better agents to treat, and perhaps even cure, epilepsy continues.

Epilepsy is a common health problem in Bangladesh. 30 to $40 \%$ of patients are still treated by traditional healer. Superstitious belief is a stigmata in the community. The most common cause of non-compliance is cost of drug. 50 to $60 \%$ patients remain symptoms free with 4 common drugs. It is estimated that there are at least 1.5 to 2.0 million epilepsy patients in Bangladesh. Epidemiological study was conducted at Epilepsy Clinic, Neurology foundation Hospital, Dhaka, Bangladesh, and a total of 2200 patients were included. Causes for treatment failure include improper compliance, incorrect diagnosis, inappropriate drug, dose \& duration, and lastly Refractory epilepsy. Causes for non-compliance (20\%) include non-affordability of drugs, unavailability of drugs, inadequate knowledge and attitude, lack of counseling, adverse effects of drug, social factors.

[Journal of Science Foundation 2018;16(2):32-33]

\section{References}

Ahmed S, Alam ST, Rahman MM, Akhter S. Clinical profile of Early Childhood Epilepsy: A Cross Sectional Study In A Tertiary Care Hospital. Mymensingh Med J. 2016;25(1):96-101

Borovicka M. Editorial: The complexity of epilepsy. Mental Health Clinician: November 2012;2(5):106-106

Habib M, Khan SU, Hoque MA, Mondal MB, Hasan AH, Chowdhury RN, et al. Antiepileptic drug utilization in Bangladesh: experience from Dhaka Medical College Hospital. BMC Research Notes 2013;6(1):473

Hussain E. Burden of Epilepsy in Bangladesh: Current Approach J Curr Adv Med Res 2017;4(1):1-2

International Bureau for Epilepsy (IBE). Epilepsy in Bangladesh. https://www.ibe-epilepsy.org/epilepsy-in-bangladesh/

Mannan MA. Epilepsy in Bangladesh. Neurol Asia. 2004;9(1):18

Mian MF, Jobayer M, Afroz Z, Chowdhury AH, Chowdhury RN, Habib M, et al. Demographic profiles of epileptic patients and their awareness towards epilepsy with the influence on compliance. Bangladesh Medical Journal. 2016;45(1):20-4 